\title{
NON-SELF-DUAL YANG-MILLS CONNECTIONS WITH NONZERO CHERN NUMBER
}

\author{
LORENZO SADUN AND JAN SEGERT
}

We prove the existence of non-self-dual Yang-Mills connections on $S U(2)$ bundles over the standard four-sphere, specifically on all bundles with second Chern number not equal to \pm 1 . A YangMills (YM) connection $A$ is a critical point of the YM action

$$
S(A)=\int_{S^{4}}|F|^{2} d \mathrm{Vol}=\int_{S^{4}}-\operatorname{Tr}(* F \wedge F),
$$

where $F$ is the curvature of the connection $A$ and $*$ is the Hodge dual. The YM equations $D * F=0$, where $D$ denotes the covariant exterior derivative, are the variational equations of this functional, and constitute a system of second-order PDE's in $A$. Absolute minima of the $\mathrm{YM}$ action, in addition to satisfying the YM equations, also satisfy a first-order system of PDE's, the (anti)self-duality equations $* F= \pm F$. We call a connection non-self-dual (NSD) if it is neither self-dual $(* F=F)$ nor antiself-dual $(* F=-F)$, i.e., if it is not a minimum of the $\mathrm{YM}$ action.

(Anti) self-dual connections on $S^{4}$ have been well-understood for some time. The first nontrivial example, the BPST instanton [BPST], was found in 1975, and three years later all self-dual solutions on $S^{4}$ were classified [ADHM], not only for $S U(2)$ but for all classical groups. The study of self-dual $S U(2)$ connections on other four-manifolds led to spectacular progress in topology, including the discovery of fake $\mathbf{R}^{4}$ (see [FU] for an overview).

The study of NSD YM connections has proceeded much more slowly. While some examples of NSD YM connections on fourmanifolds are known [I, Ma1, Ma2, Ur, P], until recently NSD YM connections on $S U(2)$ bundles over standard $S^{4}$ proved elusive,

Received by the editors April 3, 1990 and, in revised form, August 13, 1990.

1980 Mathematics Subject Classification (1985 Revision). Primary 81E13; Secondary 34B $15,53 \mathrm{C} 05,58 \mathrm{E} 30$.

The first author is partially supported by NSF Grant DMS-8806731.

The second author is partially supported by a Bantrell Fellowship and NSF Grant DMS-8801918. 
and it was widely believed that none existed. Analogies with harmonic maps of $S^{2}$ to $S^{2}$ [AJ] appeared to support nonexistence. Further, weakly stable NSD YM connections were ruled out [BLS, T1], as were NSD YM connections with a certain symmetry $[\mathrm{T} 2, \mathrm{JT}]$.

The existence of NSD YM connections on the trivial $S U(2)$ bundle over $S^{4}$ has been recently proven by Sibner, Sibner, and Uhlenbeck [SSU]. In this paper we show that NSD YM connections exist on all $S U(2)$ bundles over $S^{4}$ with second Chern number $C_{2} \neq \pm 1$. We do not know whether any exist for $C_{2}= \pm 1$. The NSD YM connections we obtain on the trivial bundle $C_{2}=0$ are different from those of [SSU]. The details of our construction will appear elsewhere.

We study connections that are equivariant with respect to a symmetry group $G=S U(2)$, that acts on $S^{4} \subset \mathbf{R}^{5}$ via the unique irreducible representation. The principal orbits are three-dimensional, reducing the YM equations and self-duality equations to systems of ordinary differential equations [Ur, BoMo]. The inequivalent lifts of this $G$ action to $S U(2)$ bundles over $S^{4}$, which we call quadrupole bundles [ASSS1], are naturally classified by a pair of odd positive integers $\left(n_{+}, n_{-}\right)$. Our main result is:

Theorem 1. On every quadrupole bundle $\left(n_{+}, n_{-}\right)$with $n_{ \pm} \neq 1$ (i.e. $n_{+} \neq 1$ and $n_{-} \neq 1$ ), there exists a non-self-dual $S U(2)$ Yang-Mills connection.

The second Chern number of a quadrupole bundle $\left(n_{+}, n_{-}\right)$is given by $C_{2}=\left(n_{+}^{2}-n_{-}^{2}\right) / 8$ [SS, ASSS1], from which we deduce:

Corollary 2. On every $S U(2)$ bundle over the four sphere with $C_{2} \neq$ \pm 1 , there exists at least one non-self-dual Yang-Mills connection.

In particular, the number of distinct non-self-dual YM connections we obtain on the bundle with a given second Chern number $C_{2}$ is equal to the number of distinct odd positive factors of $C_{2}$ (not necessarily prime), minus one if $\left|C_{2}\right|=k(k+1) / 2$ for some integer $k$. For example, $C_{2}=-45$ has six positive odd factors, $1,3,5,9,15,45$, and furthermore $45=9(9+1) / 2$, so the number of distinct solutions we obtain is five. For the trivial bundle $C_{2}=0$, we obtain the existence of countably many distinct solutions, which are of different symmetry type than the countably many solutions of [SSU]. 
Computer approximations of a number of non-self-dual solutions indicate to high accuracy that distinct solutions with the same Chern number do not have the same action, suggesting:

Conjecture 3. The moduli space of NSD YM connections is not pathconnected if $\left|C_{2}\right| \neq 3$ and $\left|C_{2}\right| \neq 2^{k}$ for any integer $k$.

There are nonequivariant moduli that come from conformal transformations of equivariant solutions, and there may be additional nonequivariant moduli, but we believe that there are no equivariant moduli.

The study of the YM equations on the quadrupole bundles of [ASSS1, SS, ASSS2] was initiated by Gil Bor and Richard Montgomery [BoMo]. Some of the ideas presented in this paper were developed in collaboration with Bor and Montgomery. We have also benefitted from discussions with Percy Deift, Lesley Sibner, Robert Sibner, Barry Simon, Cliff Taubes, and particularly Jalal Shatah.

\section{EQUIVARIANT CONNECTIONS}

We now examine the symmetry group action on quadrupole bundles.

Lemma 1.1. Let $G=S U(2)$ act on $S^{4} \subset \mathbf{R}^{5}$ via the unique irreducible representation. The principal orbits are three-dimensional, and there are two exceptional orbits of dimension two. There exists a closed geodesic segment of length $\pi / 3$ that intersects each G-orbit exactly once.

The proof and further discussion is given in [ASSS1]. The affine parameter $\theta \in[0, \pi / 3]$ parametrizes the space of orbits, the endpoints corresponding to the exceptional orbits.

The quadrupole bundles are a family of $S U(2)$ bundles over $S^{4}$ that have a natural lift of this $G$-action. These bundles are indexed $^{1}$ by two positive odd integers $\left(n_{+}, n_{-}\right)$, as we now explain. A point $x \in S^{4}$ that lies on an exceptional (i.e. twodimensional) $G$-orbit is invariant under a subgroup $U(1) \subset G$, which acts by automorphism on the fiber over $x$. This action of $U(1)$ on $G=S U(2)$ has a corresponding positive odd integer index. The two indices $\left(n_{+}, n_{-}\right)$correspond to the two distinct exceptional orbits.

\footnotetext{
${ }^{1}$ Comparing notation with [ASSS1], $n_{+}=2\left|m_{T}\right|$, and $n_{-}=2\left|m_{B}\right|$.
} 
Bor and Montgomery [BoMo] have applied Urakawa's theory of dimensional reduction [Ur] to the principal quadrupole bundles ${ }^{2}$, obtaining:

Lemma 1.2 ([BoMo]). The set of smooth equivariant connections on the quadrupole bundle $\left(n_{+}, n_{-}\right)$is in 1-1 correspondence with triplets of smooth functions $a=\left(a_{1}, a_{2}, a_{3}\right), a_{i}: S^{1} \rightarrow \mathbf{R}$ satisfying the boundary conditions $a_{3}(0)=n_{+}, a_{2}(\pi / 3)=n_{-}$, and the matching conditions

$$
\begin{gathered}
a_{1}(x)=(-1)^{\left(n_{+}+1\right) / 2} a_{2}(-x) ; \quad a_{3}(x)=a_{3}(-x) \\
a_{1}(\pi / 3+x)=(-1)^{\left(n_{-}+1\right) / 2} a_{3}(\pi ; 3-x) ; \\
a_{2}(\pi / 3+x)=a_{2}(\pi / 3-x) .
\end{gathered}
$$

If $n_{+}>1$ we also require $a_{1}(0)=a_{2}(0)=0$ and if $n_{-}>1$ we require $a_{1}(\pi / 3)=a_{3}(\pi / 3)=0$.

The space of equivariant connections on $\left(n_{+}, n_{-}\right)$is thus affine, simplifying the analysis. Because of the matching conditions (1.1), it is sufficient to specify the functions $a_{i}$ on the interval $[0, \pi / 3]$.

The curvature $F$ of an equivariant connection can be directly expressed in terms of the functions $a_{i}$. Given a choice of metric, the Yang-Mills action $S$ and the (anti)self-duality equations follow. We always use the standard homogeneous metric on $S^{4}$. For equivariant connections,

$$
\begin{aligned}
S=\pi^{2} \int_{0}^{\pi / 3} d \theta\left[\left(a_{1}^{\prime}\right)^{2} G_{1}+\left(a_{2}^{\prime}\right)^{2} G_{2}+\left(a_{3}^{\prime}\right)^{2} G_{3}\right. \\
\left.+\frac{\left(a_{1}+a_{2} a_{3}\right)^{2}}{G_{1}}+\frac{\left(a_{2}+a_{1} a_{3}\right)^{2}}{G_{2}}+\frac{\left(a_{3}+a_{1} a_{2}\right)^{2}}{G_{3}}\right],
\end{aligned}
$$

where the functions $G_{i}$, which reflect the choice of metric, are

$$
\begin{aligned}
& G_{1}(\theta)=2 \sin (\theta) \sin (\pi / 3-\theta) / \sin (\pi / 3+\theta) \\
& G_{2}(\theta)=2 \sin (\theta) \sin (\pi / 3+\theta) / \sin (\pi / 3-\theta) \\
& G_{3}(\theta)=2 \sin (\pi / 3+\theta) \sin (\pi / 3-\theta) / \sin (\theta) .
\end{aligned}
$$

By the principle of symmetric criticality $[\mathrm{Pa}]$, an equivariant connection that is stationary with respect to equivariant variations is stationary with respect to all variations. The full YM

\footnotetext{
${ }^{2}$ There are some notational differences, our $a_{1}, a_{2}, a_{3}$ being, up to sign, [BoMo]'s $a_{3}, a_{2}, a_{1}$, respectively. The sign of two of the $a_{i}$ 's can be reversed by a change of basis in the Lie algebra. We fix the signs by setting $n_{ \pm}>0$, while $\left[\right.$ BoMo] use $n_{ \pm} \equiv 1(\bmod 4)$.
} 
equations for equivariant connections are easily obtained as the Euler-Lagrange equations of (1.2). The (anti)self-dual equations are directly computed,

$$
\begin{gathered}
-a_{1}^{\prime}= \pm \frac{\left(a_{1}+a_{2} a_{3}\right)}{G_{1}}, \quad-a_{2}^{\prime}= \pm \frac{\left(a_{2}+a_{1} a_{3}\right)}{G_{2}}, \\
-a_{3}^{\prime}= \pm \frac{\left(a_{3}+a_{1} a_{2}\right)}{G_{3}}
\end{gathered}
$$

where the plus signs give self-duality and the minus signs give antiself-duality.

\section{EXISTENCE OF MINIMA}

We now sketch the proof of our main theorem. We first prove:

Proposition 2.1. On every quadrupole bundle $\left(n_{+}, n_{-}\right)$with $n_{ \pm} \neq$ 1 , there exists an equivariant Yang-Mills connection.

Note that this does not imply the main theorem, since the YM connection could be (anti)self-dual. To prove Proposition 2.1, we use the direct variational method.

We define a Hilbert space that contains the smooth equivariant connections with finite action. Let $\mathscr{H}$ be the completion of the space of triplets $b=\left(b_{1}, b_{2}, b_{3}\right)$ of smooth functions on $[0, \pi / 3]$ with respect to the norm

$$
\|b\|^{2}=\int_{0}^{\pi / 3} d \theta\left[\left(b_{1}^{\prime}\right)^{2} G_{1}+\left(b_{2}^{\prime}\right)^{2} G_{2}+\left(b_{3}^{\prime}\right)^{2} G_{3}+\frac{\left(b_{1}\right)^{2}}{G_{1}}+\frac{\left(b_{2}\right)^{2}}{G_{2}}+\frac{\left(b_{3}\right)^{2}}{G_{3}}\right] .
$$

This is the direct sum of three weighted Sobolev spaces, one for each $b_{i}$. If the functions $G_{i}$ and $1 / G_{i}$ were uniformly bounded on the interval, each would just be the ordinary Sobolev space $W_{1}^{2}$, which in one dimension embeds compactly into $C([0, \pi / 3])$, the space of continuous functions with the uniform norm. However, we can still prove a weaker Sobolev-type lemma:

Lemma 2.2. $\mathscr{H}$ embeds continuously into $C([0, \pi / 3])$.

For $n_{ \pm} \neq 1$, we let $\mathscr{H}\left(n_{+}, n_{-}\right) \subset \mathscr{H}$ consist of the functions $b$ with boundary conditions $b_{3}(0)=n_{+}, b_{2}(\pi / 3)=n_{-}$. This corresponds via Lemma 1.2 to all (continuous) equivariant connections on $\left(n_{+}, n_{-}\right) . \mathscr{H}\left(n_{+}, n_{-}\right)$is a weakly closed affine subspace of $\mathscr{H}$. 
Lemma 2.3. For any $n_{ \pm} \neq 1$ and any constant $M$, the set of connections on the quadrupole bundle $\left(n_{+}, n_{-}\right)$with action $S \leq M$ is bounded in the $\mathscr{H}$-norm (2.1).

The main difficulty in proving Lemma 2.3 comes from the singular behavior of the $G_{i}$ and $1 / G_{i}$ at the endpoints, which complicates the bounding of nonderivative terms. To control this behavior, we need the condition $n_{ \pm} \neq 1$. The derivative terms in the norm are easily bounded by the corresponding terms in the action.

Since bounded action means bounded $\mathscr{H}$-norm, and since a closed bounded subset of a Hilbert space is weakly compact by the Banach-Alaoglu theorem, a minimizing sequence of connections on $\left(n_{+}, n_{-}\right)$has a weakly convergent subsequence. Using Lemma 2.2 and the weak closure of $\mathscr{H}\left(n_{+}, n_{-}\right)$, we show that the weak limit $a^{*}$ of such a subsequence is a continuous equivariant connection on $\left(n_{+}, n_{-}\right)$.

To show that $a^{*}$ attains the minimum action, we need:

Lemma 2.4. The Yang-Mills action is weakly lowersemicontinuous on $\mathscr{H}\left(n_{+}, n_{-}\right)$.

The proof of Lemma 2.4 uses the compact embedding theorem on subintervals $[\delta, \pi / 3-\delta]$, the positivity of the action integrand, and a limiting argument.

Thus $a^{*}$ minimizes the $\mathrm{YM}$ action among equivariant connections on $\left(n_{+}, n_{-}\right)$. By the principle of symmetric criticality, it is a weak solution of the four-dimensional YM equations. Combining elliptic regularity arguments with direct analysis of the endpoint behavior, we find that $a^{*}$ is in fact a smooth strict solution of the Yang-Mills equations.

To complete the proof of Theorem 1 we need:

Proposition 2.5. On quadrupole bundles $\left(n_{+}, n_{-}\right)$with $n_{ \pm} \neq 1$, there exist no equivariant (anti) self-dual connections.

This is proved by studying the positivity properties of the first order ODE system (1.3). We show that anti-self-dual solutions cannot satisfy the boundary conditions of Lemma 1.2 if $n_{+} \neq 1$. We show that $a_{1}$ and $a_{2}$ must have the same leading-order behavior near zero, and in particular must have the same sign. Since $a_{3}(0)=n_{+}>0, a_{3}$ is of course positive near zero. By (1.3), this makes $a_{3}^{\prime}$ positive, and makes $a_{1}^{\prime}$ and $a_{2}^{\prime}$ have the same sign as $a_{1}$ 
and $a_{2}$. Thus the signs continue for all $x$, making $a_{3}(\pi / 3)$ positive (hence nonzero) and violating the boundary conditions. We similarly show that a self-dual solution cannot satisfy the boundary conditions if $n_{-} \neq 1$. (If $n_{+}$or $n_{-}=1$, the boundary conditions are not as strict, and (anti)self-dual solutions are indeed possible.)

Combining Propositions 2.1 and 2.5 completes the proof of Theorem 1.

\section{REFERENCES}

[ADHM] M. F. Atiyah, V. G. Drinfeld, N. J. Hitchin, and Y. I. Manin, Construction of instantons, Phys. Lett. 65A (1978), 185.

[AJ] M. F. Atiyah and J. D. S. Jones, Topological aspects of Yang-Mills theory, Comm. Math. Phys. 61 (1978), 97.

[ASSS1] J. E. Avron, L. Sadun, J. Segert, and B. Simon, Chern numbers, quaternions, and Berry's phases in Fermi systems, Comm. Math. Phys. 124 (1989), 595.

[ASSS2] __ Topological invariants in Fermi systems with time-reversal invariance, Phys. Rev. Lett. 61 (1989), 1329.

[BLS] J. P Bourguignon, H. B. Lawson, and J. Simons, Stability and gap phenomena for Yang-Mills fields, Proc. Nat. Acad. Sci. U.S.A. 76 (1979), 1550; Stability and isolation phenomena for Yang-Mills equations, Comm. Math. Phys. 79 (1982), 189.

[BoMo] G. Bor and R. Montgomery, SO(3) invariant Yang-Mills fields which are not self-dual, Proceedings of the MSI Workshop on Hamiltonian Systems, Transformation Groups, and Spectral Transform Methods, Montreal, Canada, October 1989.

[BPST] A. A. Belavin, A. M. Polyakov, A. S. Schwartz, and Yu. Tyupkin, Pseudoparticle solutions of the Yang-Mills equations, Phys. Lett. B59 (1975), 85.

[FU] D. Freed and K. Uhlenbeck, Instantons and four-manifolds, SpringerVerlag, New York, 1984.

[I] M. Itoh, Invariant connections and Yang-Mills solutions, Trans. Amer. Math. Soc. 267 (1981), 229.

[JT] A. Jaffe and C. Taubes, Vortices and monopoles, Birkhäuser, Boston, 1980.

[Ma1] Yu. Manin, New exact solutions and cohomology analysis of ordinary and supersymmetric Yang-Mills equations, Proc. Steklov Inst. Math. 165 (1984), 107.

[Ma2] _ Gauge field theory and complex geometry, Springer-Verlag, Berlin, 1988.

[P] T. Parker, Unstable Yang-Mills fields, preprint; Non-minimal Yang-Mills fields and dynamics, preprint.

[Pa] R. S. Palais, The principle of symmetric criticality, Comm. Math. Phys. 69 (1979), 19.

[SS] L. Sadun and J. Segert, Chern numbers for fermionic quadrupole systems, J. Phys. A 22 (1989), L111. 
[SSU] L. M. Sibner, R. J. Sibner, and K. Uhlenbeck, Solutions to Yang-Mills equations which are not self-dual, Proc. Nat. Acad. Sci. U.S.A. 86 (1989), 8610.

[T1] C. H. Taubes, Stability in Yang-Mills theories, Comm. Math. Phys. 91 (1983), 235.

[T2] - On the equivalence of the first and second order equations for gauge theories, Comm. Math. Phys. 75 (1980), 207.

[Ur] H. Urakawa, Equivariant theory of Yang-Mills connections over Riemannian manifolds of cohomogeneity one, Indiana Univ. Math. J. 37 (1988), 753.

Courant Institute of Mathematical Sciences, New York University, 251 Mercer Street, New York, New York 10012

Division of Physics, Mathematics, and Astronomy, California Institute of Technology, 114-36, Pasadena, California 91125

Current address : Department of Mathematics, University of Missouri, Columbia, Missouri 65211 